\title{
Phylogenetic and Gene Sequence Analysis of Clostridium Perfringens Genes (Cpa, Cpb, Etx and Iap) Isolated from Clinical Mastitis in Cattle Dairy Farms
}

\author{
Abeer S. El-Maghraby ${ }^{1 *}$, Elham F. El-Sergany ${ }^{2}$, Marwa M Ahmed ${ }^{2}$ and Hala ElSawy Ahmed El Sawy ${ }^{2}$
}

${ }^{1}$ Central Laboratory for Evaluation of Veterinary Biologics (CLEVB), Agriculture Research Center (ARC), Egypt

${ }^{2}$ Veterinary Serum and Vaccine Research Institute (VSVRI), Agriculture Research Center (ARC), Egypt

DOI: $10.36347 /$ sjavs.2020.v07i08.003

| Received: 02.08.2020 | Accepted: 10.08.2020 | Published: 16.08.2020

*Corresponding author: Abeer S. El-Maghraby

\section{Abstract}

The objective of study is to investigate both the phylogenetic and identification of highly conserved domains of Clostridium perfringens genes ( $c p a, c p b$, etx and iap) isolated from dairy cattle clinically infected with mastitis using bioinformatics approach to explore more information about (cpa, cpb, etx and iap) proteins and applying insilico analysis for designing potential B-epitope subunit vaccine against $C$. Perfringens type A in dairy cattle farms. Five isolates were confirmed to be $C$. Perfringens type A in a prevalence of $7.14 \%(5 / 70)$. The multiplex PCR revealed the presence of the $C P A$ gene ( $\alpha$ toxin) of a approximate size of $402 \mathrm{bp}$ while the $C P B$ gene ( $B$ toxin), ETX gene ( $\varepsilon$ toxin) and IAP gene (iota toxin) went undetected. Two conserved domains regions in the nucleotide sequence of $C$. Perfringens type A (CPA) protein were detected Zn_dep_PLPC (Zinc dependent phospholipase C-alpha toxin) (63356 bp) and Zn_dep_PLPC (Zinc dependent phospholipase C-alpha toxin) (69-356 bp). Phylogenetic analysis of $C P A$ gene showed a high identity (99-99.5\%) for $C$. perfringens type A ASM strain with $C$. perfringens type A strains present in GeneBank. The insilico study was used to identify peptide fragments from Alpha toxin of $C$. perfringens type A that can be efficiently used for the designing and development of B-epitope based vaccine. B-cell epitopes are predicted using integrated computational tools. IEDB server was used to predict B-cell epitopes on the basis of different essential parameters like antigenicity, allergenicity, surface accessibility and flexibility. Based on the results interpretation, the top peptide sequences were (SQKGTAG, NSQKGTA, QKGTAGY, KGTAGYI) obtained as potential B-cell epitopes.

Keywords: Clostridium perfringens type A, Conserved domain, cpa gene, immunoinformatic, Insilico analysis, mastitis, Multiplex PCR.

Copyright @ 2020: This is an open-access article distributed under the terms of the Creative Commons Attribution license which permits unrestricted use, distribution, and reproduction in any medium for non-commercial use (NonCommercial, or CC-BY-NC) provided the original author and source are credited.

\section{INTRODUCTION}

Bovine mastitis is a major disease affecting dairy cattle worldwide. It remains a major global challenge to milk production even in the face of wide spread implementation of mastitis control strategies [1, 2]. Despite the significant advances in understanding the disease, both clinical and sub-clinical mastitis remain a problem in dairy herds. Mastitis is a global problem as it adversely affects animal health, quality of milk and economics of milk production and every country including developed ones suffer huge financial losses [3, 4]. Mastitis usually occurs primarily in response to intramammary bacterial infection, but also to intramammary mycoplasmal, fungal, or algal infections. The severity of the inflammation can be classified into sub-clinical, clinical and chronic forms, and its degree is dependent on the nature of the causative pathogen and on the age, breed, immunological health and lactation state of the animal. Mastitis can be caused by over 250 different contagious and environmental microorganisms such as Staphylococcus aureus, Streptococcus agalactiae (both of which are contagious), Escherichia coli, Streptococci and Enterococci. Several other pathogens have been isolated in mastitic cattle mammary glands. These include Actinomyces pyrogenes, Clostridium perfringens and other organisms, such as Pseudomonas aeruginosa, Klebsiella pneumonia and Pasteurella haemolytica, Brucella melitensis, Corynebacterium bovis, Enterobacter aerogenes, Klebsiella oxytoca, Klebsiella neumoniae, Mycoplasma (various species), Proteus spp., Prototheca wickerhamii (achlorophyllic algae), Prototheca zopfii (achlorophyllic algae), Pseudomonas aeruginosa [5]. Clostridium perfringens 
is an important pathogen known to cause disease in humans and animals [6].

Clostridium perfringens type A has been recorded as being associated with many bovine mastitis-related incidents [7]. C. perfringens has the potential to produce disease on its own or to predispose the udder to the disease caused by environmental pathogens [7]. Pathogenesis of $C$. perfringensassociated infections is largely attributed to the wide array of toxins this species can produce, with $>20$ exotoxins currently identified $[8,9]$. The pathogenicity of this bacterium is largely attributable to its ability to produce a variety of virulence factors. These virulence factors include well-characterized pathogenic toxins and hydrolytic enzymes [10]. The virulence factors of $C$. perfringens can be classified functionally as membrane damaging enzymes, pore-forming toxins, intracellular toxins, and hydrolytic enzymes [9].

C. perfringens strains possess the gene encoding for phospholipase $\mathrm{C}$ (plc) (also referred to as alpha toxin $\alpha$ ) in combination with differential expression of 3 major toxin-encoding genes (beta $\beta$, epsilon $\varepsilon$, and iota 1 ) used to classify strains as toxinotypes A to E [11]. Toxin production varies among $C$. perfringens strains and is the basis for the classification system, which is based on the production of four major toxins, namely $C P A, C P B, E T X$, and ITX that divide $C$. perfringens strains into toxinotypes A to $\mathrm{E}$ [12]. The alpha toxin is the major pathogenicity factor in gas gangrene.

The coding gene (cpa) is chromosomally located, highly-conserved, and therefore present in all $C$. perfringens $[13,14]$. The genes $c p b$ (encoding CPBtoxin), cpb2 (CPB2-toxin), etx (ETX-toxin), ia/ib (ITXtoxin), are located on large plasmids of variable size [15].

$C P A$ is encoded by the cpa gene that is located on the chromosome and is produced by all strains of $C$. perfringens. $C P A$ is an enzyme that breaks down phosphatidylcholine and sphingomyelin which damages membrane phospholipids and leads to the lysis of cells. CPA also cause hemolysis, activates the arachidonic cascade and inflammation cascade which induce vasoconstriction. $C P A$ is the main toxin believed to cause gas gangrene, a disease involving extensive local tissue damage and necrosis [15]. CPA is encoded by the cpa gene that is located on the chromosome and is produced by all strains of $C$. perfringens. $C P A$ is an enzyme that breaks down phosphatidylcholine and sphingomyelin which damages membrane phospholipids and leads to the lysis of cells. $C P A$ also cause hemolysis, activates the arachidonic cascade and inflammation cascade which induce vasoconstriction. $C P A$ is the main toxin believed to cause gas gangrene, a disease involving extensive local tissue damage and necrosis [16].
Bioinformatics tools have enabled the capability of selecting potential epitopes without running the risks involved in cultivating the pathogen of interest. This kind of methodology represents a huge advantage over conventional vaccinology techniques, including faster outputs and lower costs. The concept of peptide vaccines is based on identification and chemical synthesis of B-cell and T-cell epitopes which are immunodominant and can induce specific immune responses. Well characterized protective epitopes designed from this protein can be a great help for offering consistant, cost effective and quality therapeutics against this pathogen [17].

The main aim of this study is phylogenetic and gene sequence analysis of Clostridium perfringens genes (cpa, cpb, etx and iap) isolated from clinical mastitis in cattle dairy farms and using bioinformatics approach to explore more information about (cpa, $c p b$, etx and iap) proteins and identification of highly conserved domains in Clostridium perfringens (cpa, $c p b$, etx and iap) genes sequences and applying insilico analyses for vaccine design determining potential vaccine targets against $C$. Perfringens type $\mathrm{A}$ in cattle dairy farms.

\section{MATERIALS AND METHODS Milk samples and Case defintion}

No. of 70 pooled milk samples were collected from 70 cows (each sample collected from 4 quarters of each cow) suffering from clinical mastitis (the most observed clinical manifestations were oedematous swelling of the affected quarter). The affected quarter was bluish-black in colour, with a clear line of demarcation between the necrotic gangrenous tissue and healthy tissue. The affected skin area was cold to the touch and tended to peel off, with oozing of a serous fluid, while the milk was usually bloody and watery. Samples were screened from 3 dairy farms in Giza, Qalioubia, Sharkia. The lactating cows were clinically examined for the manifestations of general clinical signs associated with udder were done according to methods described by [18]. The milk samples were collected in accordance to standards milk sampling methods described by [19].

\section{Isolation and identification of $\boldsymbol{C}$. perfringens}

The randomly collected milk samples were centrifuged at 3,000 revolutions per minute for $20 \mathrm{~min}$. The cream and supernatant were discarded, and then the sediment of each milk sample was inoculated into cooked meat broth (CMB). The samples were cultured aerobically and anaerobically, as described. After an overnight anaerobic incubation at $37^{\circ} \mathrm{C}$, using anaerobic jars containing 95\% $\mathrm{H} 2$ and 5\% CO2, a loopful was streaked onto sheep blood agar plates, containing 150 $\mu \mathrm{g} / \mathrm{ml}$ neomycin sulphate, and incubated aerobically at $37^{\circ} \mathrm{C}$ for a further $24 \mathrm{~h}$ [20]. Suspected pure colonies were identified according to morphological characters using Gram's stain, hemolysis on blood agar (double 
zone), lecithinase activity and biochemically using methods described by [21]. Nagler's reaction and toxinantitoxin test was performed according to methods described by [22]. Mice lethality test was carried out according to methods described by [23]. Dermonecrotic test was carried out according to methods described by [24].

\section{Molecular identification by Multiplex PCR}

DNA extraction. DNA extraction from samples was performed using the QIAamp DNA Mini kit (Qiagen, Germany, GmbH) with modifications from the manufacturer's recommendations. Briefly, $200 \mu 1$ of the sample suspension was incubated with $10 \mu \mathrm{l}$ of proteinase $\mathrm{K}$ and $200 \mu \mathrm{l}$ of lysis buffer at $56^{\mathrm{O}} \mathrm{C}$ for 10 min. After incubation, $200 \mu \mathrm{l}$ of $100 \%$ ethanol was added to the lysate. The sample was then washed and centrifuged following the manufacturer's recommendations. Nucleic acid was eluted with $100 \mu \mathrm{l}$ of elution buffer provided in the kit.

\section{Oligonucleotide Primers}

Primers used were supplied from Metabion (Germany) are listed in table (1) [25].

Table-1: Primers sequences, target genes, amplicon sizes and cycling conditions

\begin{tabular}{|c|c|c|c|c|c|c|c|}
\hline \multirow{2}{*}{$\begin{array}{l}\text { Target } \\
\text { gene }\end{array}$} & \multirow{2}{*}{$\begin{array}{l}\text { Primers sequences } \\
\qquad(5 ;-3)\end{array}$} & \multirow{2}{*}{$\begin{array}{l}\text { Amplified } \\
\text { segment } \\
\text { (bp) }\end{array}$} & \multirow{2}{*}{$\begin{array}{l}\text { Primary } \\
\text { denaturation }\end{array}$} & \multicolumn{3}{|c|}{ Amplification ( 35 cycles) } & \multirow{2}{*}{$\begin{array}{c}\text { Final } \\
\text { extension }\end{array}$} \\
\hline & & & & $\begin{array}{l}\text { Secondary } \\
\text { denaturath } \\
\text { on }\end{array}$ & Annealing & Extension & \\
\hline \multirow{2}{*}{$\begin{array}{l}\text { Alpha } \\
\text { toxin }\end{array}$} & GTTGATAGCGCAGGACATGTTAAG & 402 & \multirow{8}{*}{$\begin{array}{l}94^{\circ} \mathrm{C} \\
5 \mathrm{~min}\end{array}$} & \multirow{8}{*}{$\begin{array}{c}94^{\prime C} \mathrm{C} \\
30 \mathrm{sec} .\end{array}$} & \multirow{8}{*}{$\begin{array}{c}55^{\prime} \mathrm{C} \\
40 \mathrm{sec}\end{array}$} & \multirow{8}{*}{$\begin{array}{c}72^{\prime} \mathrm{C} \\
45 \mathrm{sec}\end{array}$} & \multirow{8}{*}{$\begin{array}{c}72^{\circ} \mathrm{C} \\
10 \mathrm{~min}\end{array}$} \\
\hline & CATGTAGTCATCTGITCCAGCATC & & & & & & \\
\hline Beta toxin & ACTATACAGACAGATCATTCAACC & 236 & & & & & \\
\hline \multirow[b]{3}{*}{$\begin{array}{l}\text { Epsilon } \\
\text { toxin }\end{array}$} &  & & & & & & \\
\hline & ACTGCAACIACTACTCATACTGTG & \multirow[b]{2}{*}{541} & & & & & \\
\hline & CTGGTGCCTIAATAGAAAGACTCC & & & & & & \\
\hline \multirow[t]{2}{*}{ Iota toxin } & GCGATGAAAAGCCTACACCACTAC & \multirow[t]{2}{*}{317} & & & & & \\
\hline & GGTATATCCTCCACGCATATAGTC & & & & & & \\
\hline
\end{tabular}

\section{Multiplex PCR amplification}

Primers were utilized in a $50-\mu$ reaction containing $25 \mu \mathrm{l}$ of EmeraldAmp Max PCR Master Mix (Takara, Japan), $1 \mu$ l of each primer of 20 pmol concentrations, $11 \mu \mathrm{l}$ of water, and $6 \mu \mathrm{l}$ of DNA template. The reaction was performed in an applied biosystem 2720 thermal cycler.

\section{Analysis of the PCR Products}

The products of PCR were separated by electrophoresis on $1.5 \%$ agarose gel (Applichem, Germany, GmbH) in 1x TBE buffer at room temperature using gradients of $5 \mathrm{~V} / \mathrm{cm}$. For gel analysis, $40 \mu \mathrm{l}$ of the products was loaded in each gel slot. A gelpilot 100 bp ladder (Qiagen, Gmbh, Germany) was used to determine the fragment sizes. The gel was photographed by a gel documentation system (Alpha Innotech, Biometra) and the data was analyzed through computer software.

\section{Phylogenetic, amino acids and nucleotide sequence} analysis of $C P A$ gene of $C$. Perfringens

PCR products were purified using QIAquick PCR Product extraction kit. (Qiagen, Valencia). Bigdye Terminator V3.1 cycle sequencing kit (Perkin-Elmer) was used for the sequence reaction and then it was purified using Centrisep spin column. DNA sequences were obtained by Applied Biosystems 3130 genetic analyzer (HITACHI, Japan), a BLAST® analysis (Basic Local Alignment Search Tool) [26] was initially performed to establish sequence identity to GenBank accessions. The phylogenetic tree was created by the
MegAlign module of LasergeneDNAStar version 12.1 [27] and Phylogenetic analyses was done using neighbour joining in MEGA6 [28].

Conserved Domain analysis of $\boldsymbol{C P A}$ protein sequence NCBI Search Tool was performed conserved domain analysis of the CPA protein sequence. https://www.ncbi.nlm.nih.gov/Structure/cdd/wrpsb.cgi? RID=BUR8F8WK01R\&mode=all.

\section{The physico-chemical parameters Expasy Prot} Param (http://us.expasy.org/tools/protparam.html/) was used for evaluation of physico-chemical parameters of $C P A$ protein as molecular weight, extinction coefficient, halflife, instability index, theoretical isoelectric point (pI), grand average of hydropathy (GRAVY) and total number of positive and negative residues.

\section{Secondary and tertiary structure prediction \\ Garnier-Osguthorpe-Robson (GOR) secondary structure prediction server (https://npsa- prabi.ibcp.fr/cgi-bin/secpred_gor4.pl) was used for prediction of $C P A$ protein secondary structure. 2D topology model of $C P A$ was predicted by TMBBPred server (http://bioinformatics.biol.uoa.gr/PRED- TMBB/). Swiss-model on line software (https://swissmodel.expasy.org/interactive/GZWxdt/mo dels/) was used for modeling the three-dimensional structure of $C P A$ protein. Ramachandran plot in Swiss- model was used to validate the modeled structure to see}


the quality of the resulting stereochemical of structure of $C P A$ protein.

\section{Antigenic protein identification}

Assessment of protein and proposed epitopes antigenicity was carried out according to VaxiJenv 2.0 server (http://www.ddg-pharmfac.net/vaxijen/) [29].

\section{Assessment of protein Allergenicity}

The allergenicity of protein sequences and proposed epitopes for vaccine development was carried out according to AllerTOP (http://www.ddgpharmfac.net/AllerTop/) [30].

\section{B-cell epitope identification}

Was carried out using IEDB tools (http://tools.iedb.org/main/). IEDB tools were utilized to predict B-cell epitopes were identified on the basis of parameters like antigenicity, flexibility, hydrophilicity, prediction of linear epitope and accessibility of surface. BepiPredlinear epitope prediction analysis [31], Kolaskar and Tongaonkar antigenicity scale was applied according to methods described by [32], Emini surface accessibility prediction was conducted according to methods described by [33], Chou and Fasman Beta-Turn Prediction method was performed according to method described by [34], Karplus and Schulz flexibility prediction were carried out according to methods described by [35] and hydrophilicity were identified according to methods described by [36].

\section{RESULTS AND DISCUSSION}

Five $C$. perfringens type A strains were recovered from 70 clinical mastitic cattles and confirmed phynotypically in prevalence of $(5 / 70$, $7.14 \%$ ). All suspected $C$. perfringens strains were identified for their biochemical reactions. The results employing Nagler's reaction, toxin-antitoxin test, Mice lethality test, all the five tested $C$. perfringens isolates were recorded as toxigenic. Using the dermo-necrotic test in guinea pigs to type the $C$. perfringens strains revealed in all the five $C$. perfringens isolates were identified as $C$. perfringens type A (100\%). Our results agree with [7].

The multiplex PCR amplification of C. perfringens cpa, cpb, etx and iap genes using specific primer sequences revealed a approximate size of $402 \mathrm{bp}$ (Fig.1). Cpa ( $\alpha$ toxin) gene was found in $5(100 \%)$ isolates. perfringens type A. The multiplex PCR detected the ( $\alpha$ /cpa) gene while the $(\mathrm{B} / \mathrm{cpb}),(\varepsilon / \mathrm{etx})$ and iap genes went undetected. $C$. perfringens has the potential to produce cattle gangernous mastitis on its own or to predispose intramammary infections caused by major mastitis and environmental pathogens, our results agree with [7].

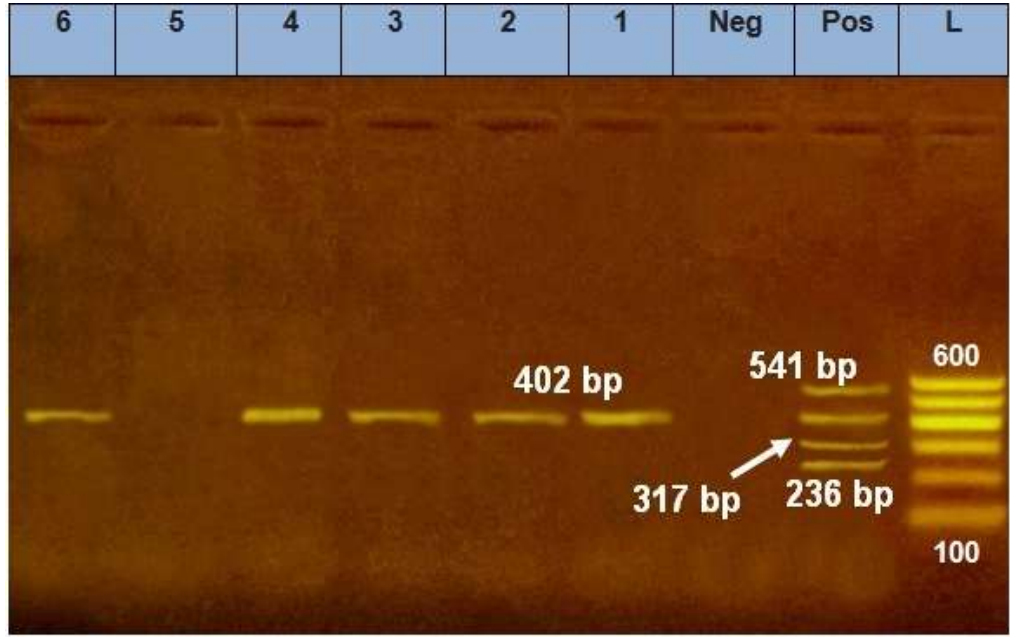

Fig-1: Agarose gel showing multiplex polymerase chain reaction (MPCR) amplified product of 402 bp of $C P A$ virulence gene for $C$. Perfringens type $A$, lanes $(1,2,3,4,6)$ : samples positive for $C P A$ gene, lane (5): samples negative for $C P A$ gene, Lane (pos.): positive control, Lane (Neg.): Negative control, Lane (L): MW 100bp ladder (DNA marker)

Putative conserved domains of $C P A$ gene of $C$. perfringens type A have been detected (Fig. 2). Most of the sequences belonged to ZnPC_SIPI super family Protein. The two conserved domains region in the nucleotide sequence are Zn_dep_PLPC (Zinc dependent phospholipase C-alpha toxin) (63-356 bp) and Zn_dep_PLPC (Zinc dependent phospholipase C-alpha toxin) (69-356 bp). 


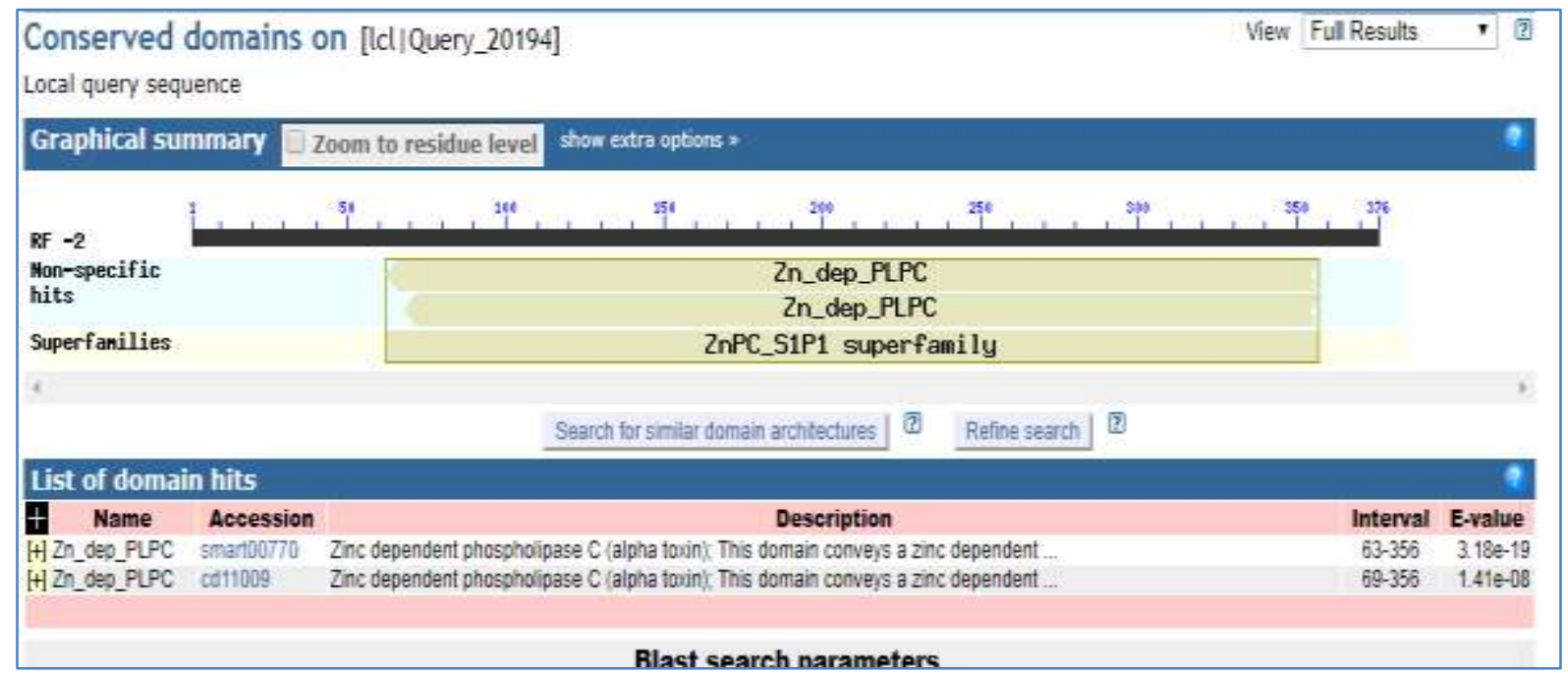

Fig-2: The 2 conserved domains region in the nucleotide sequence are Zn_dep_PLPC (Zinc dependent phospholipase C-alpha toxin) (63-356 bp) and Zn_dep_PLPC (Zinc dependent phospholipase C-alpha toxin) (69-356 bp)

Phylogenetic and partial gene sequence analysis of $C P A$ gene of $C$. perfringens type $\mathrm{A}$ that was generated using neighbor joining in MEGA6 (Fig. 3, 4), showed clear clustering and (99-99.5\%) identity with MH900556 C. perfringens CP400, GQ071566 C. perfringens LF_4a, AU605736 C. perfringens 22, DQ184152 C. perfringens NRRLB-2351, DQ184134 C. perfringens NRRLB-2351, DQ184130 C. perfringens NRRLB-2350.

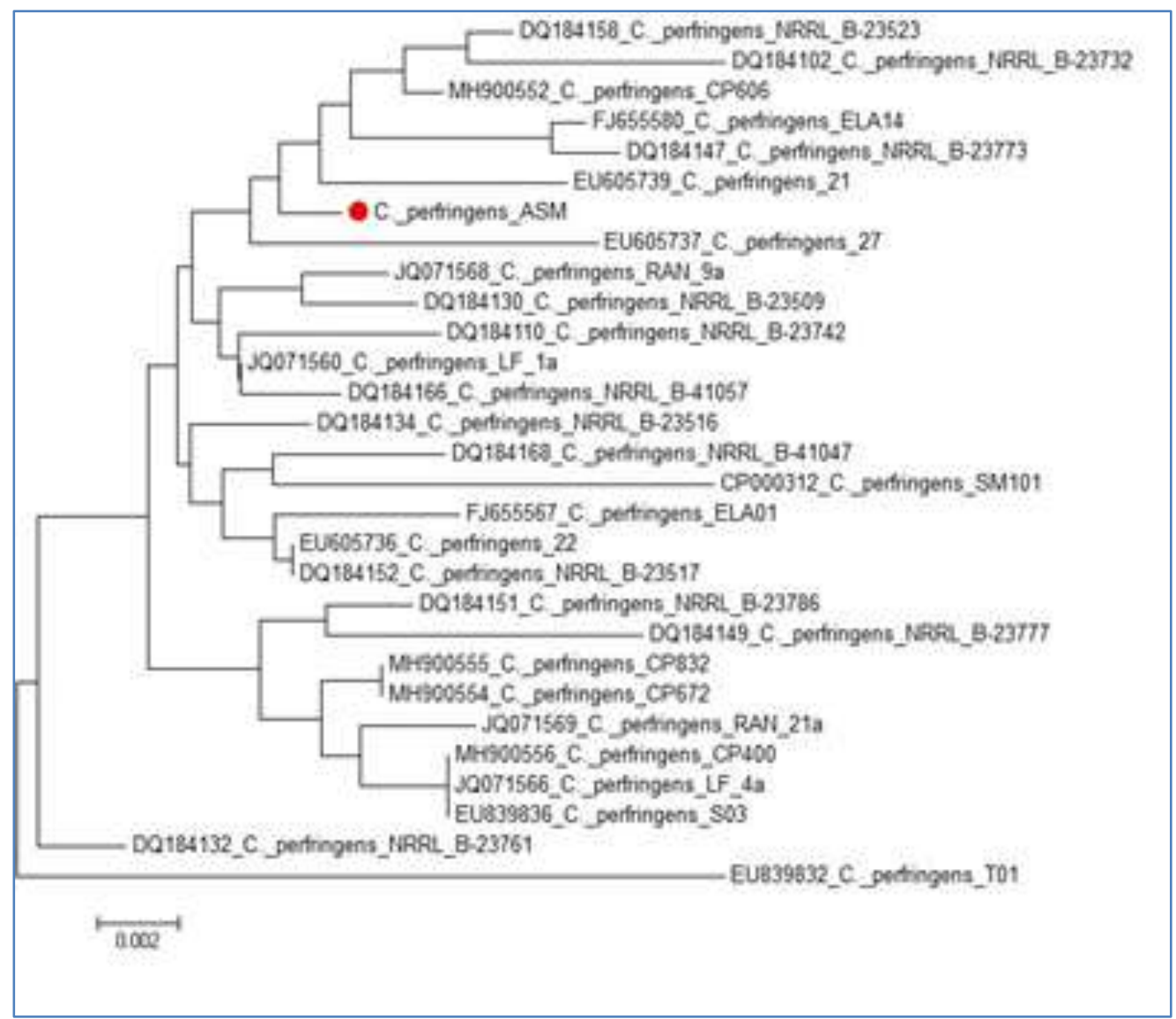

Fig-3: Phylogenetic tree of $C P A$ virulence gene for $C$. Perfringens type $\mathrm{A}$ and partial nucleotide sequences that was generated using neighbor joining in MEGA6. It showed clear clustering of the Egyptian isolated strain and different $C$. Perfringens type strains uploaded from GenBank 
Majortty

vir900556 c. perfringens cp400 $30071566 \mathrm{C}$, perfringens tf 4 a Eub39s 36 c. perfringens 503 vir900555 C, perfeingens CPB3z Yhaooss4 C. perfringens CP672 jo071569 c. perfringens RAN.21a Mig905s2 C. perftingens C $P 606$ De184158 C. perfringens NRRL B-23523 DQ184151 C. perfringens NRRL B-23780

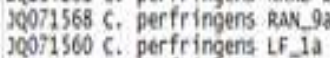

Deis4110 C. perfringens NRRL $5-23742$

00184149 C. perfringens NRRL, B-2377

DetBu166 C; perferingens NRkL B-410S? Ev605736 C. perfringens 22

De184152 C. perfringens NRRL b-2351?

00184134 C. perfringens NRRL B-23516

$00184130 \mathrm{c}$, perfeingens NRRL $\mathrm{B}-23509$

cus 39832 c. perfringens T01

F) 655567 C, perfringens ELAOL

DQ184168 C. perfringens NRQL $8=41047$

F) 655580 C. perfringens ELA14

00184132 C. perfringens NRRL $8-23761$

E0605737 c. perfeingens 27

CP000312 C. perfringens sN101

Ev605739 6 . perfringens 21

De184102 C. perfringens NRRL, $8=23732$

De184147 C, perffingens NRRL B-23773

c. perfringens $A Q N$
VDSAGPWXFETFAEERKEOWXINTAGCKTNEDFYADILKNKDENAWSXEYARGF AKTGKSTWSHASUSHSWDOWOYAAK

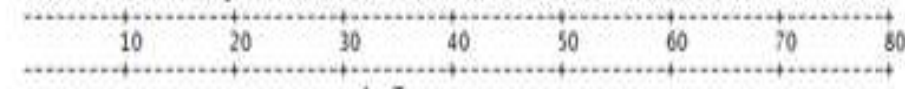

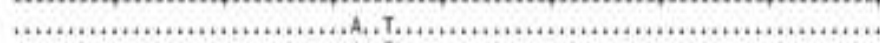

, , , , , , , , , , , , , , , , , , , ,

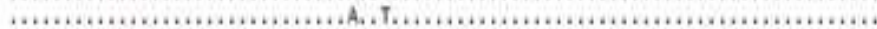

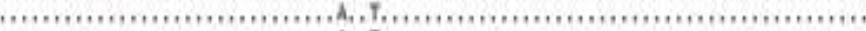

........................... . T ....................................

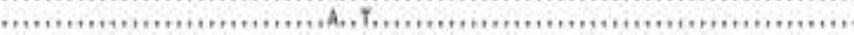

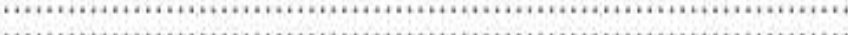

,

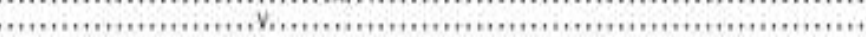

.

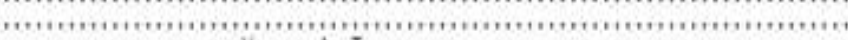

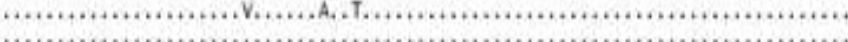

(2.

,

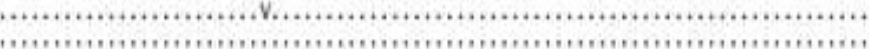

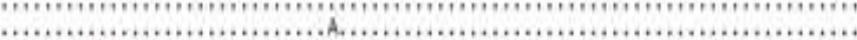

7.,

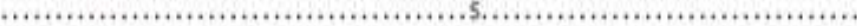

.....................

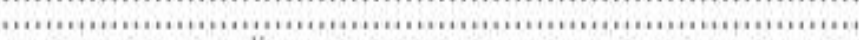

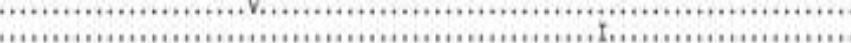

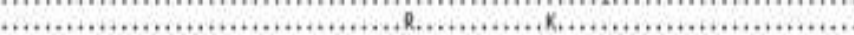

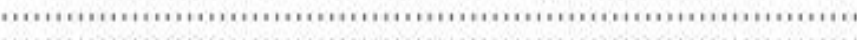

\section{8}

238

238

Majortty

VTLANSQKGTAGYTYRFLHOVSEGNOPSVGNVKELVVAYISTSGEKOAGTOON

Mw900556 C, perfringens CP\$00

X,071566 C. perfringens LE. 4 .

EuB39336 C. perfeingens 503

vih900555 C. perfringens CPB3.

Yw900554 C, perfringens $C P 672$

30071569 c, perfringers RAN_21.

Yir $900552 \mathrm{C}$, perferingens $\mathrm{C} P 606$

00184158 C. perfringens NRRL $8-23523$

oe184151 C, perfringens NRRL B-23786

jo071565 C. perfringens RAr_9a

$30071560 \mathrm{C}$. perfringens LF $1 \mathrm{a}$

$00184110 \mathrm{C}$, perfringens NRQQ B-2374?

De184149 C. perfringens NeRL $8-2377$

De184166 C, perfringens NRRL 0-4105?

Ev605736 C. perfringens 22

0.184152 C, perfringens NRRL $8-23517$

DQ184134 C. perfringens. NRRL B-23S16

Del84130 C, perfringens NRRL $8-23500$

EUB 39532 C. perfringens TO1

Fj655567 C. perfringens tLa01

00184168 C. perfringens NRQL B-4104?

F 655580 C, perfringens ELALA

Q0184132 C. perfringens NeQL B-23761

Ev605737 C, perferingens 27

Cp000312 C. perfringens sM101

Ev605739 C, perfringens 21

EU605739 C. perfringens. 21
00184102 C. perfringens NRRL $8-23732$

DQ184147 $C$, perfringens NRRL $8-23773$

c. perfringens $A S$

$\begin{array}{cccc}90 & 100 & 110 & 120\end{array}$

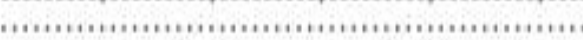

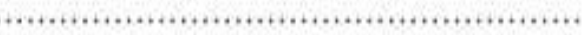

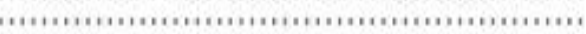

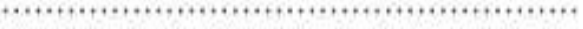

c...,

等,



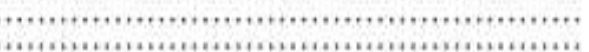

, , , , , , , , , , , , , , , , , , , , , , , , , , , , , , , , ,

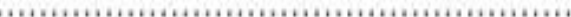

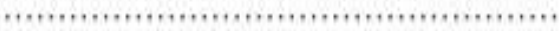



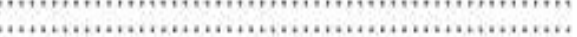

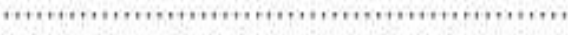

(1)

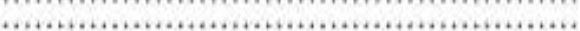

.

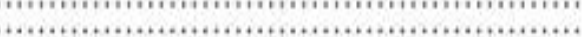

4.1,

6

-

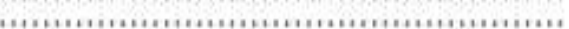



.

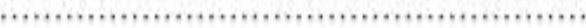

Fig-4: Deduced amino acids alignment of $C$. Perfringens type A (CPA) virulence gene of Egyptian isolated strain using CLUSTAL W multiple sequence alignment program version 1.83 of Mega Align module of laser gene DNA star and different $C$. Perfringens type a strains uploaded from GenBank

Conventional techniques for vaccine development are laborious and time consuming. As a result, computational methods [17, 37] for predicting epitopes have attracted attention of the researchers to reduce the cost and time of vaccine development to fight with the rapidly growing devastating organisms. Current immuno- informatics tools are able to predict B-cell and T-cell epitopes with high accuracy. These tools are playing a vital role in understanding the molecular basis of immunity and, notably in the development of epitope based-peptide vaccines [38]. In comparison to the conventional vaccines, peptide or epitope based vaccines are also easy to develop, chemically stable, more specific, and free of any infectious or oncogenic potential hazard [39]. 
Physical and chemical properties of the selected sequence of $C P A$ protein using Expasy ProtParam software were predicted. $C P A$ consists of 7 negatively charged residues (Asp+Glu), and 5 positively charged residues (Arg+Lys). Parameters such as molecular weight (6136.76), theoretical pI (isoelectric point) (5.39), instability index (16.67), aliphatic index (thermal stability) (70.91), and grand average of hydropathicity (-0.491) which indicate the solubility of the proteins (GRAVY) are summarized in
(Table 2). When we were analyzed the physicochemical parameters of our CPA gene by Expasy ProtParam software, the pI value (PI value 5.39) amino acids are acidic in nature. Expasy ProtParam classifies the CPA protein as stable on the basis of instability index (instability index, 16.67). Extinction coefficient of CPA protein at $280 \mathrm{~nm}$ was $16,960 / \mathrm{M} / \mathrm{cm}$ (high). The biocomputed half-life was 30 hours. Aliphatic index and Grand average of hydropathicity (GRAVY) of CPA protein were 70.91 and - 0.491, respectively.

Table-2: Physico-chemical parameters of C. Perfringens type A (CPA)

\begin{tabular}{|c|c|c|c|}
\hline Number of amino acids & \multicolumn{3}{|l|}{55} \\
\hline Molecular weight & \multicolumn{3}{|l|}{6136.76} \\
\hline Theoretical pI & \multicolumn{3}{|l|}{5.39} \\
\hline \multirow[t]{22}{*}{ Amino acid coiompositn } & Ala (A) & 5 & $9.1 \%$ \\
\hline & $\operatorname{Arg}(\mathrm{R})$ & 1 & $1.85 \%$ \\
\hline & $\operatorname{Asn}(\mathrm{N})$ & 3 & $5.5 \%$ \\
\hline & Asp (D) & 5 & $9.1 \%$ \\
\hline & Cys (C) & 0 & $0 \%$ \\
\hline & $\mathrm{Gln}(\mathrm{Q})$ & 1 & $1.8 \%$ \\
\hline & Glu (E) & 2 & $3.6 \%$ \\
\hline & Gly (G) & 4 & $7.3 \%$ \\
\hline & His $(\mathrm{H})$ & 2 & $3.6 \%$ \\
\hline & Ile (I) & 2 & $3.6 \%$ \\
\hline & Leu (L) & 3 & $5.5 \%$ \\
\hline & Lys (K) & 4 & $7.3 \%$ \\
\hline & $\operatorname{Met}(\mathrm{M})$ & 1 & $1.8 \%$ \\
\hline & Phe $(\mathrm{F})$ & 1 & $1.8 \%$ \\
\hline & Pro $(\mathrm{P})$ & 1 & $1.8 \%$ \\
\hline & $\operatorname{Ser}(\mathrm{S})$ & 6 & $10.9 \%$ \\
\hline & Thr (T) & 3 & $5.5 \%$ \\
\hline & $\operatorname{Trp}(\mathrm{W})$ & 2 & $3.6 \%$ \\
\hline & Tyr (Y) & 4 & $7.3 \%$ \\
\hline & Val (V) & 5 & $9.1 \%$ \\
\hline & Pyl (O) & 0 & $0 \%$ \\
\hline & $\operatorname{Sec}(\mathrm{U})$ & 0 & $0 \%$ \\
\hline Total number of negatively charged residues $(\mathrm{Asp}+\mathrm{Glu})$ & \multicolumn{3}{|l|}{7} \\
\hline Total number of positively charged residues (Arg +Lys) & \multicolumn{3}{|l|}{5} \\
\hline \multirow[t]{5}{*}{ Atomic Composition } & Carbon & $\mathrm{C}$ & 274 \\
\hline & Hydrogen & $\mathrm{H}$ & 410 \\
\hline & Nitrogen & $\mathrm{N}$ & 72 \\
\hline & Oxygen & $\mathrm{O}$ & 87 \\
\hline & Sulfur & $\mathrm{S}$ & 1 \\
\hline Formula/Total number of atoms & \multicolumn{3}{|c|}{$\mathrm{C}_{274} \mathrm{H}_{410} \mathrm{~N}_{72} \mathrm{O}_{87} \mathrm{~S}_{1}$} \\
\hline Extinction coefficients at $280 \mathrm{~nm}$ measured in water & \multicolumn{3}{|c|}{16960} \\
\hline Estimated half-life & \multicolumn{3}{|c|}{$\begin{array}{c}30 \text { hours (mammalian reticulocytes, in vitro). } \\
>20 \text { hours (yeast, in vivo). } \\
>10 \text { hours (Escherichia coli, in vivo). }\end{array}$} \\
\hline Instability index & \multicolumn{3}{|c|}{$\begin{array}{l}\text { This classifies the protein as stable } \\
\text { The instability index (II) is computed to be } 16.67\end{array}$} \\
\hline Aliphatic index/Grand average of hydropathicity (GRAVY) & \multicolumn{3}{|c|}{$70.91 /-0.491$} \\
\hline
\end{tabular}

The secondary structure prediction of the $C P A$ protein was $16.36 \%$ alpha helix, $27.27 \%$ extended strand, beta turn $(0.00 \%)$ and $56.36 \%$ random coil (Fig. 5a). Spoctopus servers were applied for determining membrane protein topology. No transmembrane helix found in CPA selected sequence (Fig. 5b). These results proved that our selected protein can be cloned in E.Coli host or any other bacterial host. 


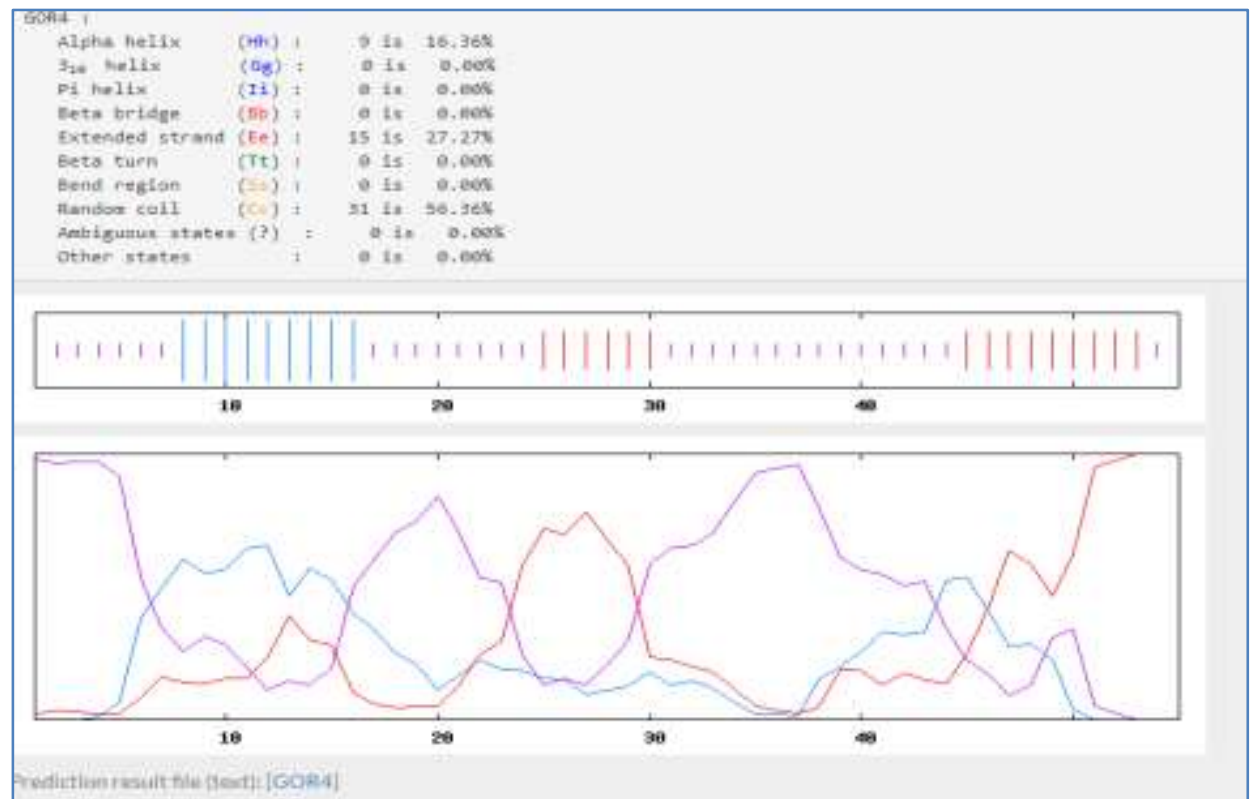

Fig-5a: Graphical results for secondary structure prediction of CPA protein composition was $16.36 \%$ alpha helix, 27.27\% extended strand, beta turn $(0.00 \%)$ and $56.36 \%$ random coil. (Purple, red, and blue colors indicate extended strand, coil, and helix, respectively)

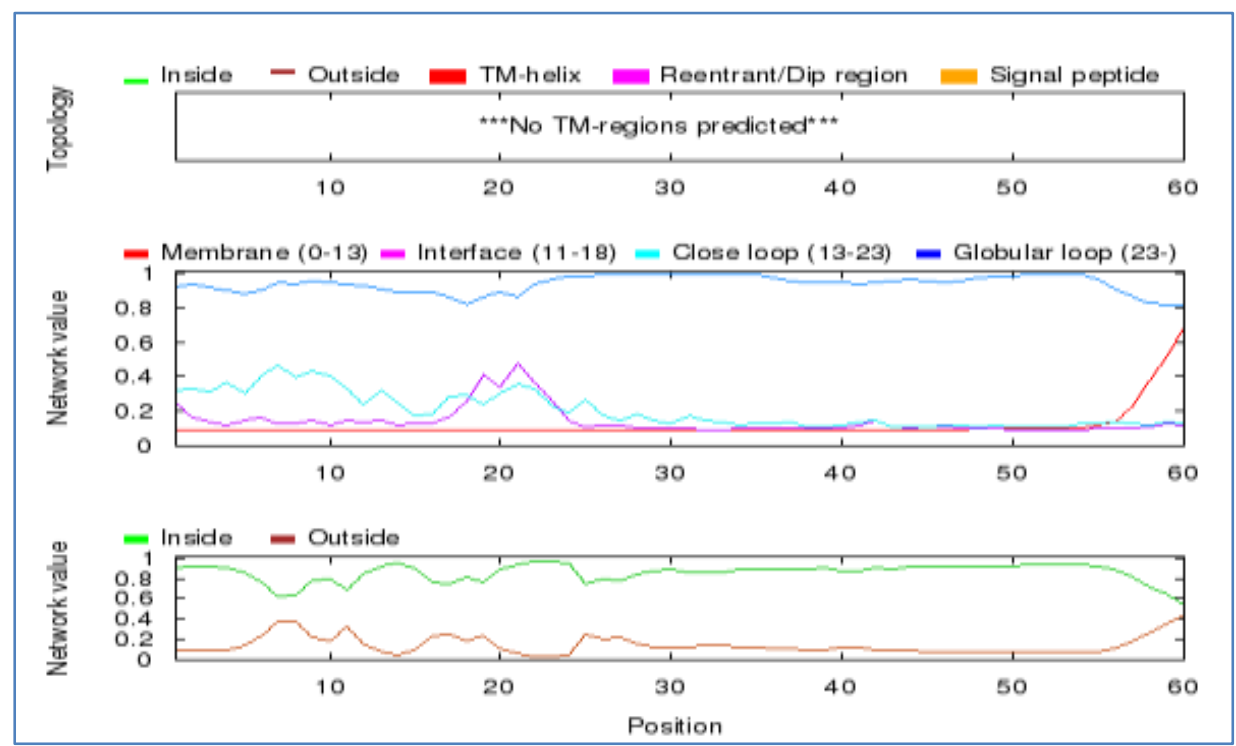

Fig-5b: SPOCTOPUS servers were applied for determining membrane protein topology. No transmembrane helix found in $C P A$ selected sequence

Swiss model was recruited for homology modeling prepared 3 different models (Fig. 6a). The identity score was $100 \%$. Our 3D structure revealed a protein with full stability based on Ramachandran plot predictions. The 3D model validity was evaluated by Ramachandran blot (Fig. 6b) where it was seen $98.11 \%$ of the residues are in favoured region, and $1.89 \%$ of the residues were in allowed region of the plot and $0.0 \%$ of residue in outlier region. The more number of residues in favoured region the more stable is the protein [40]. QMEAN is a composite scoring function for the estimation of the global and local model quality (Fig. $6 c)$. The protein model has a $Z$-score of -1.78 , this value denote higher quality of the model. GMQE (The global quality estimation of the model) is 0.98. GMQE is a quality estimation which combines properties from the target-template alignment and the template search method. The resulting GMQE score is expressed as a number between 0 and 1 , reflecting the expected accuracy of a model built with that alignment and template and the coverage of the target. The QMEAN $\mathrm{Z}$-score in the figure provides an estimate of the "degree of nativeness" of the structural features observed in the model on a global scale. QMEAN Z-scores around zero indicate good agreement between the model structure and experimental structures of similar size. Scores of 4.0 or below are an indication of models with low quality. The "Local Quality" plot (Fig. 6c) shows, for each residue of the model (reported on the x-axis), the expected similarity to the native structure (y-axis). Typically, residues showing a score above 0.6 are expected to be of high quality. 


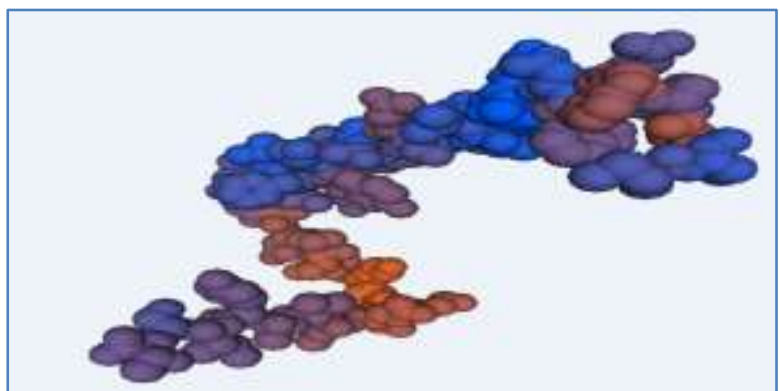

Fig-6a: The final 3D structure model of CPA (phospholipase C-protein) obtained after homology modeling by Swiss-Model

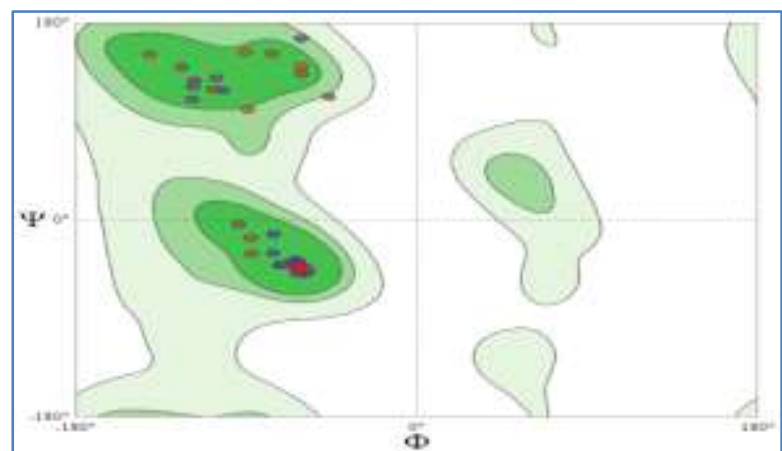

Fig-6b: Validation of protein structure using Ramachandran plot. The Ramachandran plot shows that $98.11 \%$ of amino acid residues from modeled structure were incorporated in the favored regions of the plot. $1.89 \%$ of the residues were in allowed regions of the plot and $0.0 \%$ of residue in outlier region

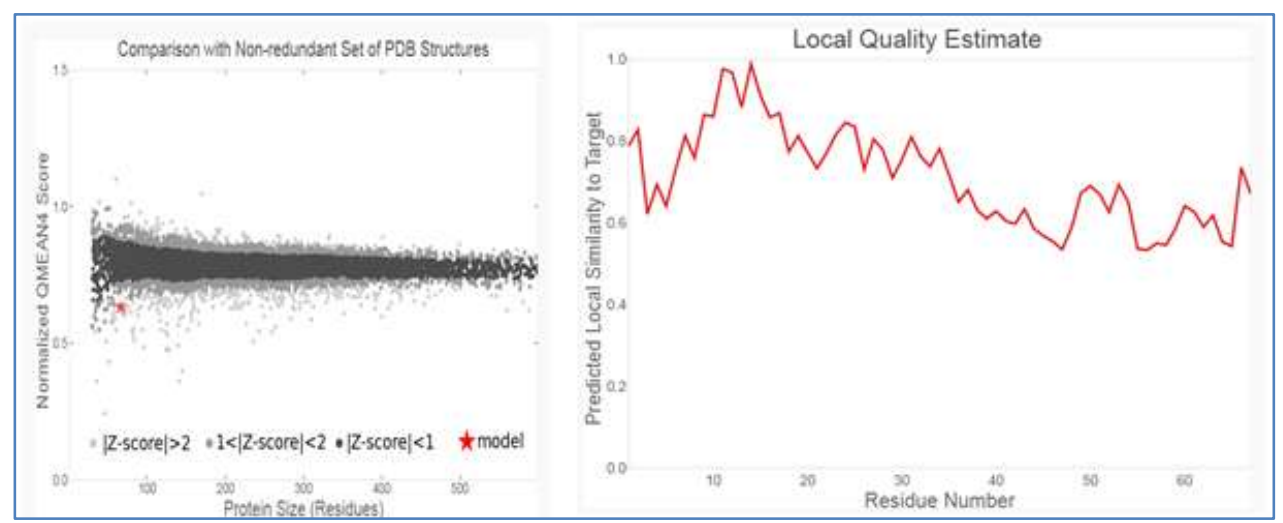

Fig-6c: Model validations. Both global and local estimation of the quality of the obtained model are reasonable. QMEAN is a composite scoring function for the estimation of the global and local model quality. The score of a model is also shown in relation to a set of high-resolution PDB structures (Z-score). Our model has a Z-score of $\mathbf{- 1 . 7 8}$ which referred to higher quality of the model

VaxiJen server was used to predict antigenic properties of the protein. The threshold value for those models provided by VaxiJen server was 0.4 . The server predicted CPA protein of $C$. perferingens type $\mathrm{A}$ as antigenic having an overall prediction score of 0.8 . The overall prediction score of predicted top 4 B-cell Epitopes were antigenic (more than 0.4) (Table 3). In silico analysis revealed that all selected protein sequence and all 4 predicted top B-cell epitopes in this study were non-allergenic in nature. Allergenicity is one of the prominent obstacles in vaccine development since today most vaccines stimulate the immune system into an "allergic" reaction [17]. Allergenicity prediction of selected protein sequence of all 4 predicted top Bcell epitopes was done using Allertop (Table 3) and it was predicted that there were no evidences about the specific peptide sequences arousing any allergic reactions into the host body. This confirmed our selected conserved sequence CPA protein and peptides to be potentially immunogenic.

Table-3: List of the best predicted scored B-cell epitopes of final vaccine.

\begin{tabular}{|c|c|c|c|c|l|}
\hline Epitope sequence & Residue & Start & End & Vaxijen Score & Aller-top \\
\hline SQKGTAG & $\mathrm{G}$ & 19 & 25 & 2.5 & Non Allergen \\
\hline NSQKGTA & $\mathrm{K}$ & 18 & 24 & 1.97 & Non Allergen \\
\hline QKGTAGY & $\mathrm{T}$ & 20 & 26 & 1.59 & Non Allergen \\
\hline KGTAGYI & $\mathrm{A}$ & 21 & 27 & 1.26 & Non Allergen \\
\hline
\end{tabular}


In order to predict a peptide that can be used in vaccine development to prevent $C$. perfringens type A infection in cattle dairy farms, this study focused on CPA protein of $C$. perfringens type A. Multiple prediction methods were applied to determine a potential B-cell epitope considering several criteria like Bepipred Linear Epitope Prediction (Fig. 11), antigenicity (Fig. 7), beta turn prediction (Fig. 9), allergenicity, surface accessibility (Fig. 8), hydrophilicity (Fig. 12) and flexibility (Fig. 10). The proposed four epitopes (SQKGTAG, NSQKGTA, QKGTAGY, KGTAGYI) have met all the criteria of the above B-cell prediction methods.

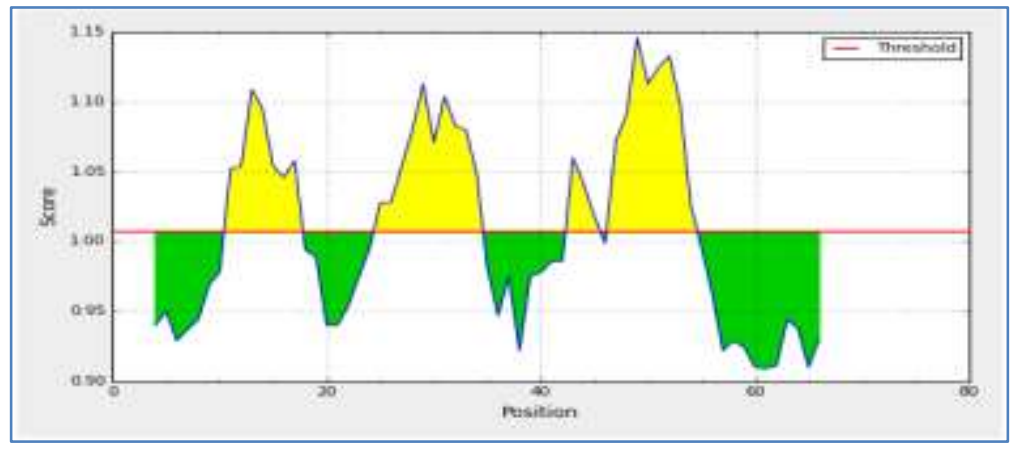

Fig-7: Graph obtained from Kolaskar and Tongaonkar Antigenicity method

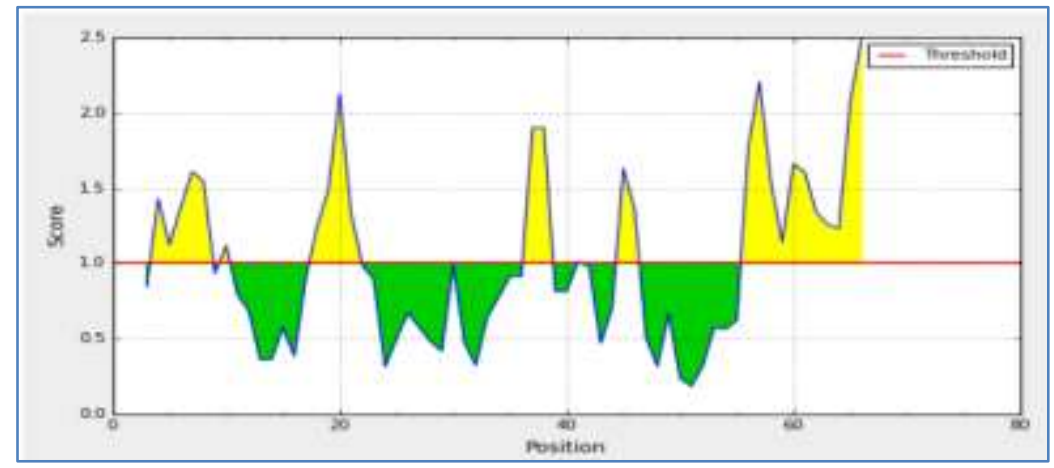

Fig-8: Graph obtained from Emini Surface Accessibility Prediction Results

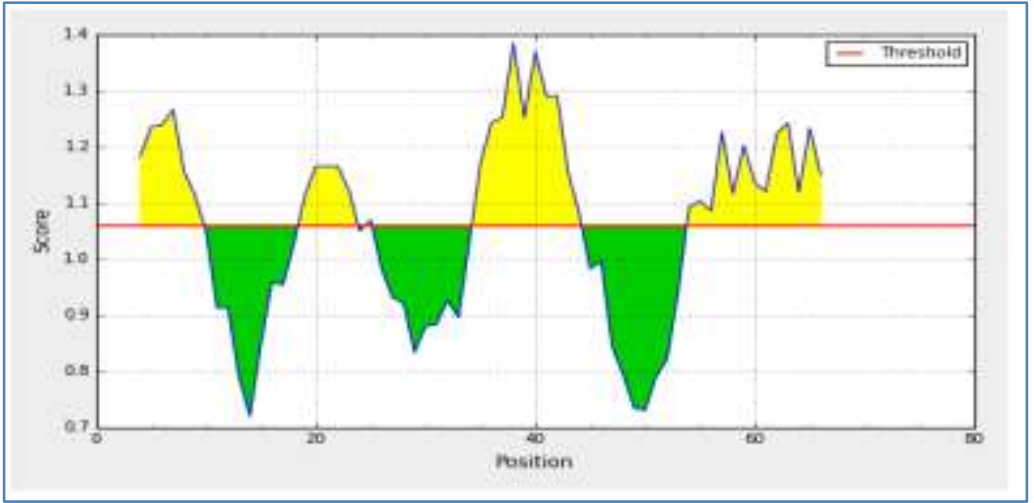

Fig. 9: Graph obtained from Chou \& Fasman Beta-Turn Prediction method

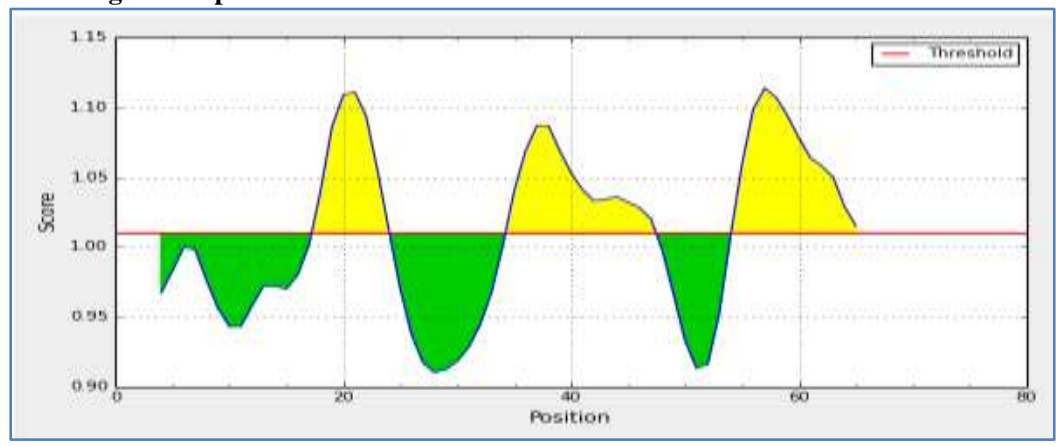

Fig-10: Graph obtained from Karplus \& Schulz Flexibility Prediction method 


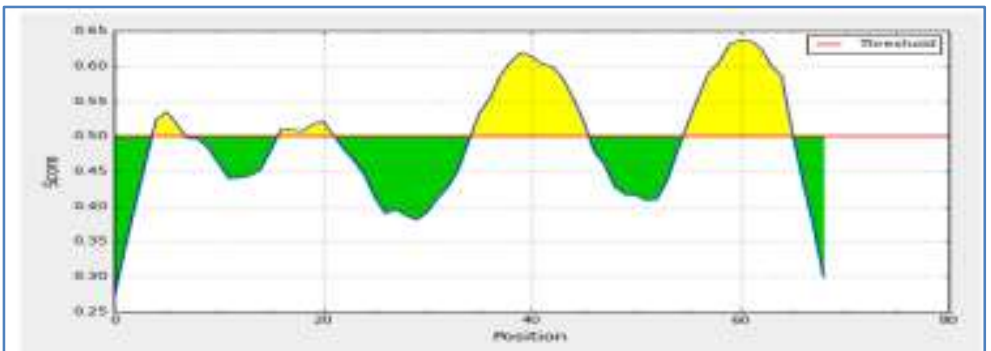

Fig-11: Graph obtained from Bepipred Linear Epitope Prediction 2.0 through IDEB tool

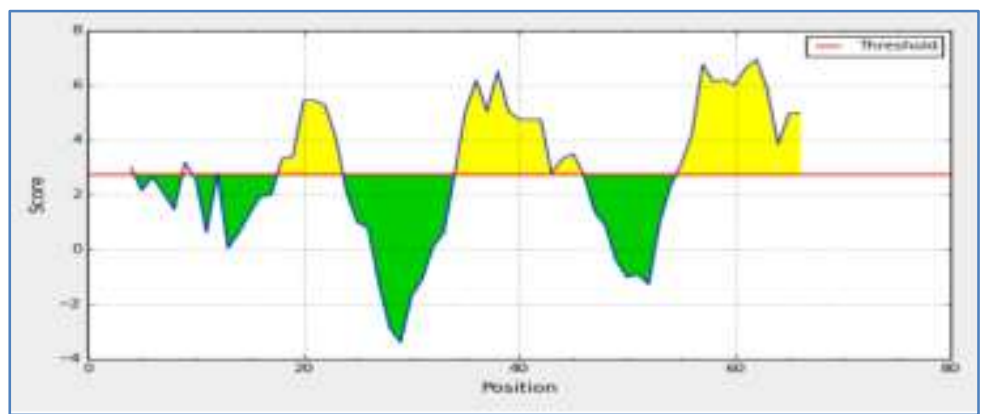

Fig-12: Graph obtained from from Parker Hydrophilicity Prediction

\section{REFERENCES}

1. Skuce PJ, Bartley DJ, Zadoks RN, Macleod M. Livestock health and green house gas emissions. Climate X Change is Scotland's Centre of Expertise on Climate Change. 2016, pp: 1-74. Available at: https://sefari.scot/sites/default/files/documents/Sku ce\%2CP.\%20.

2. Getaneh AM, Mekonnen SA, Hogeveen $H$. Stochastic bio-economic modeling of mastitis in Ethiopian dairy farms; Prev. Vet. Med. 2017, 138: 94-103.

3. Hogeveen H, Van der Voort M. Assessing the economic impact of an endemic disease: the case of mastitis; Rev. Sci. Tech. Off. Int. Epiz. 2017, 36 (1): 217-226. doi: 10.20506/rst.36.1.2623.

4. Hogeveen H, Steeneveld W, Christopher A. Wolf. Production diseases reduce the efficiency of dairy production: A review of the results, Methods, and approaches regarding the economics of mastitis; Annu. Rev. Resour. Econ. 2019, 11: 289-312.

5. Bhuvana M, Shome BR. Etiology of bovine mastitis model training course on "Bovine mastitis: Theoretical and practical consideration in management $1^{\text {st }}$ to $8^{\text {th }}$. 2013, Available on line at:https://krishi.icar.gov.in/jspui/bitstream/1234567 89/23634/1/MTC\%20Bovine\%20Mastitis\%20Trai ning\%20Manual.pdf.

6. Kiu R, Hall LJ. An update on the human and animal enteric pathogen Clostridium perfringens; Emerg. Microbes Infect. 2018, 7: 1-15.

7. Osman KM, El-Enbaawy MI, Ezzeldeen NA, Hussein HMG. Mastitis in dairy buffalo and cattle in Egypt due to Clostridium perfringens: prevalence, incidence, risk factors and costs; Rev. sci. tech. Off. int. Epiz. 2009, 28 (3): 975-986.
8. Kiu R, Caim S, Alexander S, Pachori P, Hall LJ. Probing genomic aspects of the multi-host pathogen Clostridium perfringens reveals significant pangenome diversity, and a diverse array of virulence factors; Front Microbiol. 2017, 8: 2485.

9. Revitt-Mills SA, Rood JI, Adams V. Clostridium perfringens extracellular toxins and enzymes: 20 and counting; Microbiol. Aust. 2015, 36, 114-117. doi: 10.1071/MA15039.

10. Li J, Paredes-Sabja D, Sarker MR, McClane BA. Clostridium perfringens sporulation and sporulation- associated toxin production; Microbiol. Spectr. 2016, 4: 1-27. doi: 10.1128/microbiolspec.TBS-0022-2015.

11. Omer SA, Ebtesam M. Al-Olayan, Salah-Eldin H. Babiker, Mohammed Z. Aljulaifi, Abdulaziz N. Alagaili, Osama B. Mohammed. Genotyping of Clostridium perfringens isolates from domestic livestock in Saudi Arabia; BioMed. Research International. 2020, Vol. 2020, Article ID 9035341, 9 pages. https://doi.org/10.1155/2020/9035341.

12. McClane BA, Robertson SL, Li J. Clostridium perfringens in Food Microbiology, eds M. Doyle and R. Buchanan (Washington, DC: ASM Press). 2013, 465-489. doi: 10.1128/9781555818463.ch18.

13. Freedman JC, Shrestha A, McClane BA. Clostridium perfringens enterotoxin: action, genetics, and translational applications; Toxins. 2016, 8: E73. doi: 10.3390/toxins8030073.

14. Rood JI, Adams V, Lacey J, Lyras D, McClane BA, Melville SB, Moore RJ, Popoff MR, Sarker MR, Songer JG, et al. Expansion of the Clostridium perfringens toxin-based typing scheme; Anaerobe. 2018, 53: 5-10.

15. Li J, Adams V, Bannam TL, Miyamoto K, Garcia JP, Uzal FA, et al. Toxin plasmids of Clostridium 
perfringens. Microbiol. Mol. Biol. Rev. 2013, 77: 208-233. doi: 10.1128/MMBR.00062-12.

16. Uzal FA, Vidal JE, McClane BA, Gurjar AA. Clostridium perfringens toxins involved in mammalian veterinary diseases; Open Toxinology J. 2010, 2: 24-42.

17. Tahirah Y. Prediction of B- and T-cell epitopebased peptide vaccines from highly conserved regions in enterovirus D68 capside protein VP1: a computational approach; J. of Innovations in Pharmaceuticals and Biological Sciences. 2015, 2 (4): 426-438. www.jipbs.com.

18. Quinn PJ, Carter ME, Markey B, Carter GR. Clinical Veterinary Microbiology. London Wild life Publisher. 2004, pp. 95-101.

19. National Mastitis Council (NMC). Laboratory Handbook on Bovine Mastitis, revised ed. Madison, Wisconsin: National Mastitis Counc, Inc. 1999, pp. 1.

20. Willis TA. Anaerobic bacteriology, Clinical and Laboratory Practice, $3^{\text {rd }}$ ed., 1977, Butterworths, London.

21. Quinn PJ, Markey BK, Leonard FC, Fitzpatrick ES, Fanning S, Hartigan PJ. Veterinary Microbiology and Microbial Diseases. $2^{\text {nd }}$ ed. Wiley- Blackwell Scientific Publication, Oxford UK.19. Clayton, J., 1999. Changing trends in the laboratory diagnosis of gastroenteric infections. Nurs. Stand. 2002, 14: 42- 46.

22. Willis AT, Hobbs G. Some new media for the isolation and identification of Clostridia. J. Path. Bact. 1959, 77: 511-521.

23. Holdeman LV, Cato EP, Moore WEC. Anaerobic laboratory manual, $4^{\text {th }}$ edition. Blacksburg: Anaerobic laboratory. 1977, Virginia polytechnic institiute and state university.

24. Oakley CL, Warrack GH. Routine typing of Clostridium welchii. J. hyg. 1953. 51: 102-107.

25. Yoo HS, Lee SU, Park KY, Park YH. Molecular typing and epidemiological survey of prevalence of Clostridium perfringens types by multiplex PCR. J. of Clin. Microb. 1997, 35 (1): 228-232.

26. Altschul SF, Gish W, Miller W, Myers EW, Lipmanl DJ. Basic local alignment search tool; J. Mol. Biol. 1990, 215: 403-410.

27. Thompson JD, Higgins DG, Gibson TJ. CLUSTAL $\mathrm{W}$ : improving the sensitivity of progressive multiple sequence alignment through sequence weighting, position-specific gap penalties and weight matrix choice; Nucleic Acids Research.1994, 22 (22): 4673-4680.

28. Tamura K, Stecher G, Peterson D, Filipski A, Kumar S. MEGA6: molecular evolutionary genetics analysis version 6.0; Mol. Biol. Evol. 2013, 30: 2725-2729.

29. Doytchinova IA, Flower DR. VaxiJen: a server for prediction of protective antigens, tumour antigens and subunit vaccines; Bioinformatics (BMC). 2017, 8 (1): 4. https://doi.org/10.1186/1471-2105-8-4.

30. Dimitrov I, Bangov I, Flower DR, Doytchinova I. AllerTOP v.2-a server for in silico prediction of allergens; J. Mol. Model BioMed. Central Ltd. 2014, $20 \quad$ (6): 227. DOI:https://doi.org/10.1007/s00894-014-2278-5.

31. Jespersen MCPB. BepiPred-2.0: improving sequence-based B-cell epitope prediction using conformational epitopes. Nucleic Acids Res. 2017

32. Kolaskar A, Tongaonkar PC. A semi-empirical method for prediction of antigenic determinants on protein antigens; FEBS letter. 1990, 276: 172-4.

33. Emini EA, Hughes JV, Perlow D, Boger J. Induction of hepatitis A virus-neutralizing antibody by a virus-specific synthetic peptide; J. Virol. 1985, 55: 836-9.

34. Chou P, Fasman G. Prediction of the secondary structure of proteins from their amino acid sequence; Adv. Enzymol. 1978, 47: 45-148.

35. Karplus PA, Schulz GE. Prediction of chain flexibility in proteins. Naturwissenschaften. 1985, 72: 212-213.

36. Parker J, Guo D, Hodges R. New hydrophilicity scale derived from high-performance liquid chromatography peptide retention data: correlation of predicted surface residues with antigenicity and X-ray-derived accessible sites; Biochemistry. 1986, 25: 5425-32.

37. Lafuente EM, Reche PA. Prediction of MHCpeptide binding: a systematic and comprehensive overview, Current Pharmaceutical Design. 2009, 15 (28): 3209-3220.

38. Hasan MA, Hossain M, Alam MJ. A computational assay to design an epitope-based peptide vaccine against Saint Louis Encephalitis virus; Bioinform. Biol. Insights. 2013, 7: 347-355.

39. Holland J, Domingo E. Origin and evolution of viruses; Virus Genes. 1998, 16 (1): 13-21.

40. Notredame C, Higgins DG, Herinha J. T-Coffee: Anovel method for fast and accurate multiple sequence alignment; J.M.B. 2000, 302: 205-217. 